\title{
Improving Radio Frequency Identification-Based Localization Accuracy Using Computer-Vision-Assisted Sensor Deployment Technology
}

\author{
Rong-Shue Hsiao, ${ }^{*}$ Chun-Hao Kao, Hsin-Piao Lin, and Kai-Wei Ke ${ }^{1}$ \\ Department of Electronic Engineering, National Taipei University of Technology, \\ No. 1, Sec. 3, Zhongxiao E. Rd., Taipei 10608, Taiwan, R.O.C. \\ ${ }^{1}$ Department of Computer Science and Information Engineering, National Taipei University of Technology, \\ No. 1, Sec. 3, Zhongxiao E. Rd., Taipei 10608, Taiwan, R.O.C.
}

(Received August 30, 2016; accepted January 6, 2017)

Keywords: sensor deployment, computer vision, indoor localization, RFID, genetic algorithm

Radio frequency identification (RFID) technology is one of the promising technologies enabling the realization of the Internet of Things. However, the current major application is limited to using its identification ability. The number of applications will be increased if we can enable RFID technology to have the capability of accurate localization. In this paper, we propose a new sensor deployment method for improving the passive RFID localization accuracy. The proposed method integrates computer vision technology while employing a genetic algorithm to find the appropriate locations to deploy RFID reader antennas. The proposed method was applied to a fingerprintingbased indoor localization system. The result showed that the localization accuracy can be effectively improved by selecting the appropriate deployment locations of RFID reader antennas.

\section{Introduction}

Radio frequency identification (RFID) is a wireless identification technology that can transfer the identity information stored in RFID tags (transponders) to RFID readers. The passive RFID technology with no battery guarantees the lifelong functioning of the tag. It is also easily attached to objects, which is the most widely used method in a variety of applications. ${ }^{(1,2)}$ The passive ultrahigh-frequency (UHF) RFID tag has distinctive advantages of low cost, long reading range, and identification capability, which is one of the most promising solutions for Internet of Things (IoT) realization. ${ }^{(3)}$

Since indoor location is the most important aspect of context for mobile users, such as locating objects and people, wireless indoor localization technology is highly in demand in context-aware applications. $^{(4)}$ In recent years, wireless indoor localization has become one of the promising technologies. In Refs. 5-7, the authors provide a review of wireless indoor localization with a comprehensive comparison of different technologies. Among the wireless indoor localization techniques, the received-signal-strength (RSS)-based technique is very popular. Since RSS measurement is readily available in wireless communication without additional cost, many researchers have recently utilized passive UHF RFID tags for indoor localization. ${ }^{(8-10)}$ However, 
RSS-based indoor localization suffers significantly from multipath fading, shadowing, and radio interferences in indoor environments. ${ }^{(11)}$ In addition, the limitations of passive RFID arise from the need for these tags to absorb sufficient power from the reader to transmit the stored tag data in complex indoor environments. The main factors affecting the performance of a tag response include tag/reader location and orientation, impedance mismatch between tag antenna and chip, communication blind spot, and interference (i.e., tag-to-tag, reader-to-tag, and reader-to-reader interferences). ${ }^{(12)}$ Hence, localization accuracy may drop considerably in indoor environments.

Several researchers have attempted to model the dependence of the RSS uncertainty to improve the accuracy of location estimation. ${ }^{(13,14)}$ However, even though they continuously try all possible ways of minimizing the error, the accuracy of RSS-based distance measurement can be easily affected by complex indoor environments. Hence, with the traditional radio propagation model, it is difficult to fulfill the requirements of accurate localization. Instead of relying on accurate estimations of angle or distance to derive the location with geometry, the location fingerprinting technique associates location-dependent RSS signatures to infer a location. This method has been proved to be very accurate for indoor localization. ${ }^{(15,16)}$ Therefore, location fingerprinting has become one of the most popular and extensive techniques applied to RSS-based indoor localization systems.

For localization systems, accuracy and precision are the two most important metrics of performance evaluation. The localization accuracy is determined from the error in the distance from the actual location, while the localization precision is determined from the percentage of location information which is within the error distance. ${ }^{(17)}$ These two metrics need to be considered in an indoor localization system. Actually, improving the localization accuracy means increasing the percentage of localization results within the error distance. In the localization system of location fingerprinting, the location fingerprints are collected by performing a site survey of the RSS from multiple sensor nodes. The entire target area is divided into a set of rectangular grid points. The RSS is measured to create a database of predetermined RSS values on the points of the grids. The localization accuracy depends on the grid spacing, which is closely tied to the application requirements. The localization precision depends on the number of sensor nodes. However, as the localization accuracy increases (error distance is reduced), the localization precision may decrease. ${ }^{(18)}$ Thus, there exists a trade-off between accuracy and precision of localization. Another parameter affecting localization precision is the deployment location of sensor nodes. In Refs. 19 and 20, the authors show that the larger the RSS Euclidean distance between the sensor nodes, the stronger the system tolerance to signal fluctuation and the smaller the probability of fault location in the wireless indoor localization system. We can adjust the deployment of the sensor nodes to increase the RSS Euclidean distance between the neighboring sensor nodes, so that the localization accuracy can be improved. Therefore, the deployment location of sensor nodes is a key issue for the fingerprintingbased indoor localization.

Finding the ideal deployment locations for a set of sensors in a given area is a difficult problem, which has been proven to be NP-complete. ${ }^{(21)}$ The genetic algorithm is a computational model that mimics the process of natural selection and genetics in biological evolution. The genetic algorithm has been widely used to solve the combinatorial optimization problems with complex constraints. The computation of the genetic algorithm is an iterative process that achieves the global optimum. ${ }^{(21-23)}$

In this paper, we focus on the investigation of a sensor deployment strategy for improving indoor localization accuracy. We proposed a novel deployment method, which exploits computer vision technology and a genetic algorithm to find the appropriate locations for sensor node (RFID 
reader antenna) deployment. The error of location estimation can be decreased and the localization accuracy can be improved. To solve this problem, a genetic algorithm is presented.

The remainder of the paper is organized as follows. In Sect. 2, we present our proposed sensor deployment method. The experimental setup and results are presented in Sects. 3 and 4, respectively. Finally, the conclusion is presented in Sect. 5.

\section{Materials and Methods}

In fingerprinting-based indoor localization systems, the location fingerprints are collected by performing a site survey of the RSS from multiple RFID reader antennas. Owing to the effects of radio frequency propagation, such as multipath fading, shadowing, and other interferences, the radio map of the offline phase may not be suitable for the location estimation in the online phase. In order to improve the localization accuracy and precision, we propose a computer-vision-assisted sensor deployment method to find the appropriate deployment locations of RFID reader antennas to build a proper radio map. The proposed method includes a genetic algorithm to solve the optimization problem.

\subsection{Framework of the proposed sensor deployment method}

The proposed method consists of two main components: a computer vision system and a genetic algorithm, as shown in Fig. 1. The proposed method performs in the offline stage of the fingerprinting-based localization system. The computer vision system can promptly provide actual target location. The actual location is used as ground truth for comparison with the result of fingerprinting-based localization. The comparison of these two locations is consequently used in the genetic algorithm for the evaluation of RFID reader antenna deployment. Then, a set of ideal deployment locations for a given number of RFID reader antennas is determined. The selected deployment locations of RFID reader antennas result in an appropriate radio map and localization accuracy improvement.

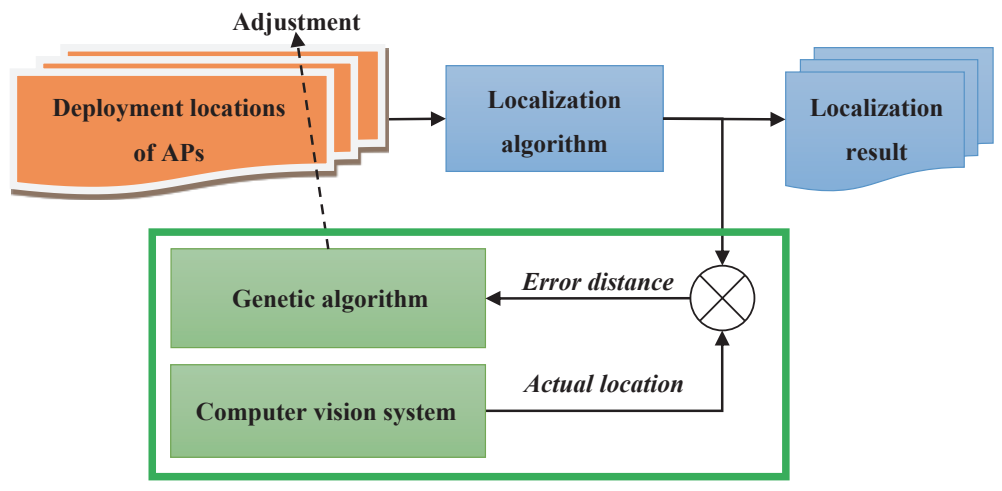

Fig. 1. (Color online) Framework of the proposed sensor deployment method. 


\subsection{Computer vision system}

The computer vision system is used to locate the target tag, which includes two steps: image acquisition and image localization. To locate the target tag in an image with a complex background, as shown in Fig. 2(a), the target tag is marked with a colored cross pattern. While conducting image acquisition, a color-depth camera is used to capture color and depth information simultaneously. Image localization includes image segmentation and coordinate transformation. Image segmentation extracts candidate blobs from the pattern by the thresholding method on the depth and color images. Depth information represents the distance from the camera to the pattern, while color information represents the color of the pattern. For the depth image, threshold values are the range of a given distance; for the color image, the threshold values are the range of given color. Subsequently, morphological image processing is used to extract the blob of the color pattern, which is the intersection of the candidate blobs, as shown in Fig. 2(b). Since the center of the blob is the location of the target tag, the location is transformed into physical coordinates. Then, the location coordinates are provided to the genetic algorithm.

\subsection{Proposed genetic algorithm}

The goal of the genetic algorithm is to find the ideal deployment locations of RFID reader antennas which result in the least localization error. In the genetic algorithm, a population contains several candidate deployment locations of reader antennas. A candidate set of deployment locations of reader antennas is called an individual. Thus, the size of the population indicates the number of individuals. A chromosome consists of various genes, which indicate the properties of an individual, such as the number of reader antennas and the range of deployment location. The length of the chromosome represents the number of genes. The location of a reader antenna, $(x, y)$, is represented by a pair of genes. The $x$ - and $y$-coordinates are bounded by the range of deployment, namely, the upper and lower boundaries. In this paper, the real-coded genetic algorithm (RGA) is adopted, so every gene is directly represented by a floating number. Hence, encoding and decoding

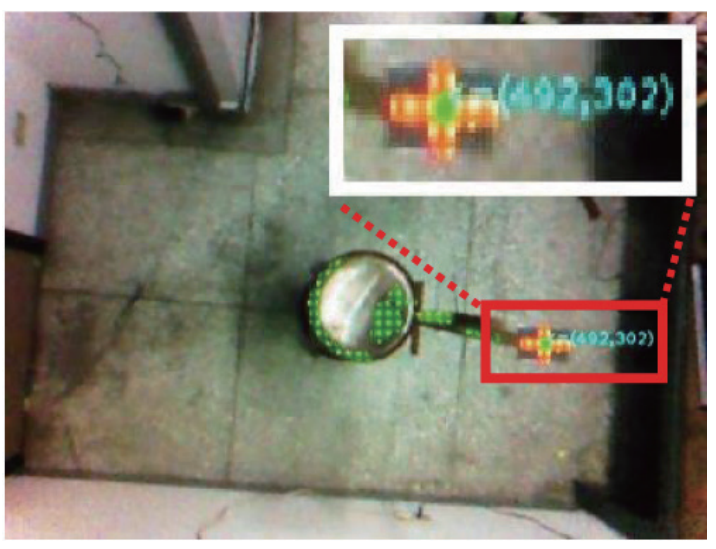

(a)

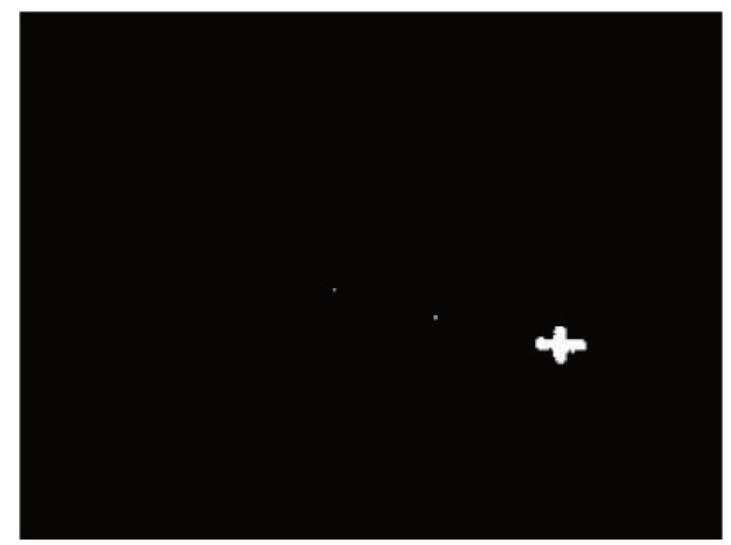

(b)

Fig. 2. (Color online) Image segmentation: (a) color image (the center of the blob is marked) and (b) blob of the extracted cross pattern. 
processes for genes are not needed. The fitness value denotes the localization error of an individual (deployment locations of reader antennas) for the deployment environment (fitness function). In this study, the localization error was determined by comparing the actual location of the target tag with the result of the localization algorithm in the real environment. It was not just evaluated using formulas in the simulated environment. The genetic algorithm solves the optimization problem, which evolves toward a better individual that has a smaller fitness value. The proposed genetic algorithm is shown in Table 1.

To generate next generation of population, there are three genetic operations: selection, crossover, and mutation. The selection operation removes individuals that have low fitness values from the population. After that, the remaining individuals are selected for performing crossover and mutation. The worst individuals are eliminated and replaced by offspring, which are recombined from several of the best individuals.

The crossover operation produces a new child (offspring) through the recombination of a pair of parents. For the RGA, a chromosome can be treated as an n-dimension real vector. Thus, the candidate individuals form an $n$-dimension real space $\left(R^{n}\right)$. According to the searching space, it can

Table 1

Proposed genetic algorithm.

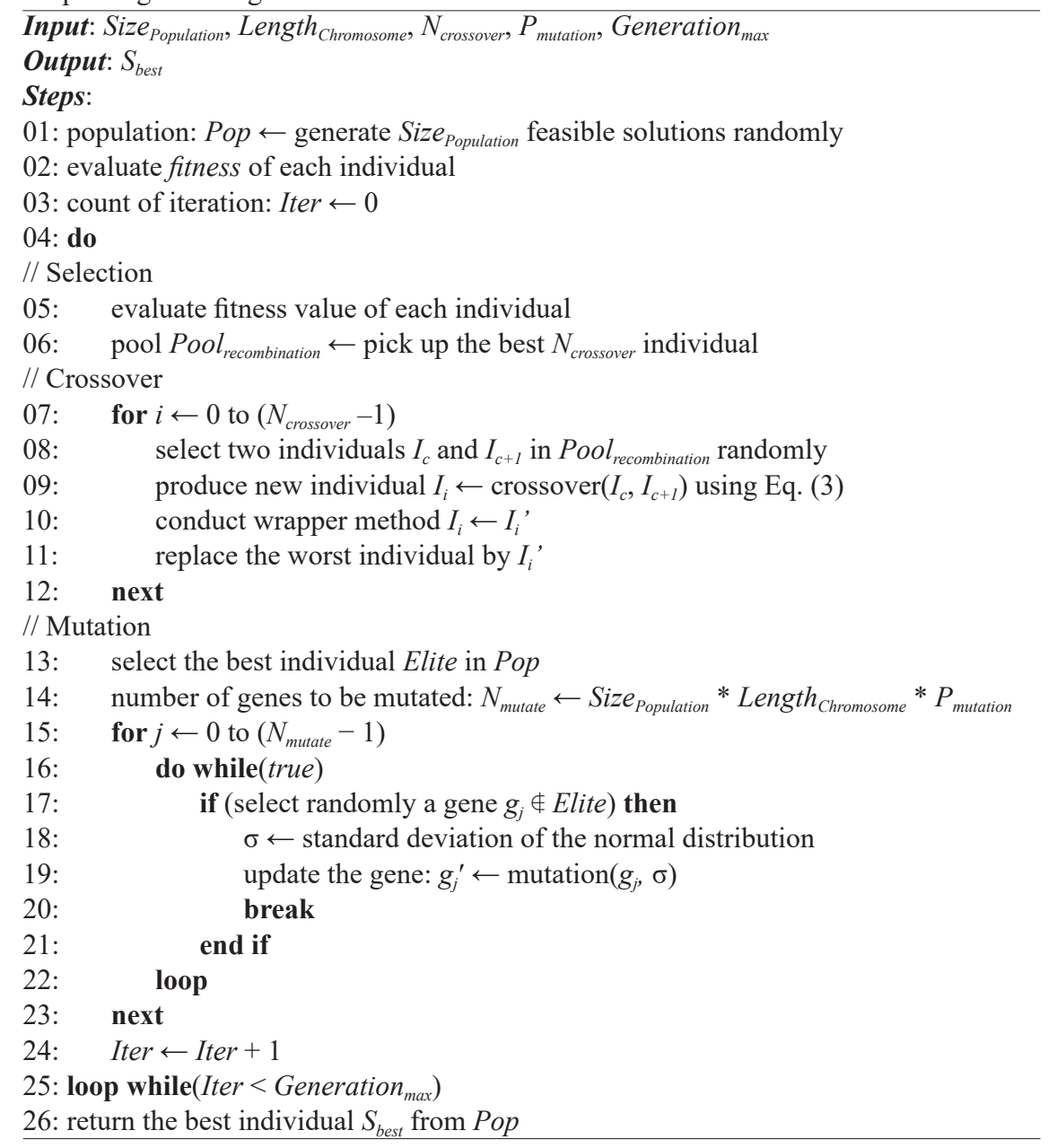


be categorized by line crossover and box crossover. The line crossover searches offspring along a vector, which is a line segment between two parents, whereas the box crossover searches offspring in a box. To produce better offspring by selecting superior parents, the conventional crossover performs the inside search method, shown in Eq. (1).

$$
p_{\text {new }}=\beta \times p_{m n}+(1-\beta) \times p_{d n},
$$

where $\beta=$ a random number in $[0,1], p_{m n}=n$th gene from parent $p_{m}$, and $p_{d n}=n$th gene from parent $p_{d}$.

However, the range of the bounded searching space is limited by the parents. It may cause premature convergence due to lower population diversity, which results in local optima. ${ }^{(22)}$ Another method, in contrast, is to search outside away from the parents, but the searching range is still limited by the parents, as follows:

$$
p_{\text {new }}=\beta \times\left(p_{m n}-p_{d n}\right)+p_{m n} .
$$

Hence, some research studies extend the searching space toward the outside of parents, including extended-line crossover and extended-box crossover. In comparison with the conventional RGA searching method, the proposed bidirectional searching method combines an inside search [Eq. (1)] and an outside search [Eq. (2)], as shown in Eq. (3).

$$
p_{\text {new }}=S I G N_{\text {Direction }} \times \beta \times\left(p_{m n}-p_{d n}\right)+p_{m n},
$$

where $S I G N_{\text {Direction }} \in\{-1,+1\}$ : searching direction, -1 : search inside, and +1 : search outside.

However, the range of gene values is discontinuous near the boundary in the bidirectional searching method. To make the searching space continuous, the value space is wrapped. Thus, the upper bound and the lower bound are glued together as a glued space (or adjunction space, quotient space). According to previous studies, ${ }^{(24,25)}$ performing crossover in a glued space can avoid the bias of offspring toward the center of the searching space. The bias of offspring may result in a boundary problem. To overcome the boundary problem, the wrapper method is performed, as shown in Table 2.

Table 2

Wrapper method.

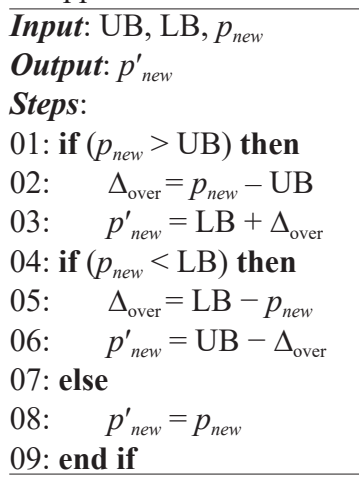


If the gene value ( $p_{\text {new }}$ ) of the offspring exceeds the upper bound (UB) or the lower bound (LB), the remainders are wrapped to another boundary. The commonly used roulette wheel method is used to decide the searching direction. The selection operator is adopted by performing a random shuffle. A larger searching area (inside or outside) is preferred because of a larger area with a higher probability. Using boundary wrapping, the experimental results showed that the higher performance of crossover results in a higher probability of finding the optimal solution.

The mutation performs a random search between the upper bound and the lower bound of a gene. It explores possible solutions other than the current solution space. Before performing mutation, the elite individual with the best fitness is preserved and is not involved in the mutation. Finally, the genetic algorithm terminates when the number of iteration reaches maximum generations $\left(\right.$ Generation $\left._{\max }\right)$.

\section{Experimental Setup}

\subsection{Experimental parameters and equipment}

To verify the effectiveness of the proposed method, we conducted an experiment in our lab, as shown in Fig. 3. In a small area $\left(2 \times 1.6 \mathrm{~m}^{2}\right)$, we deployed one RFID reader with 5 antennas for RSS measuring and locating the target tag. The target tag is a UHF passive RFID tag. For the computer vision system, a camera was installed in the center of the ceiling, which is used to monitor the whole sensing area to detect the location of a moving target. The computer vision system was used to build the radio map of a target tag with vision-based 2D location. There are 15 distinct locations of target tags and 5 candidate locations of antennas. The radio map was constructed in a person and one passive tag environment. In addition, the location of the target tag can be determined by the computer vision system.

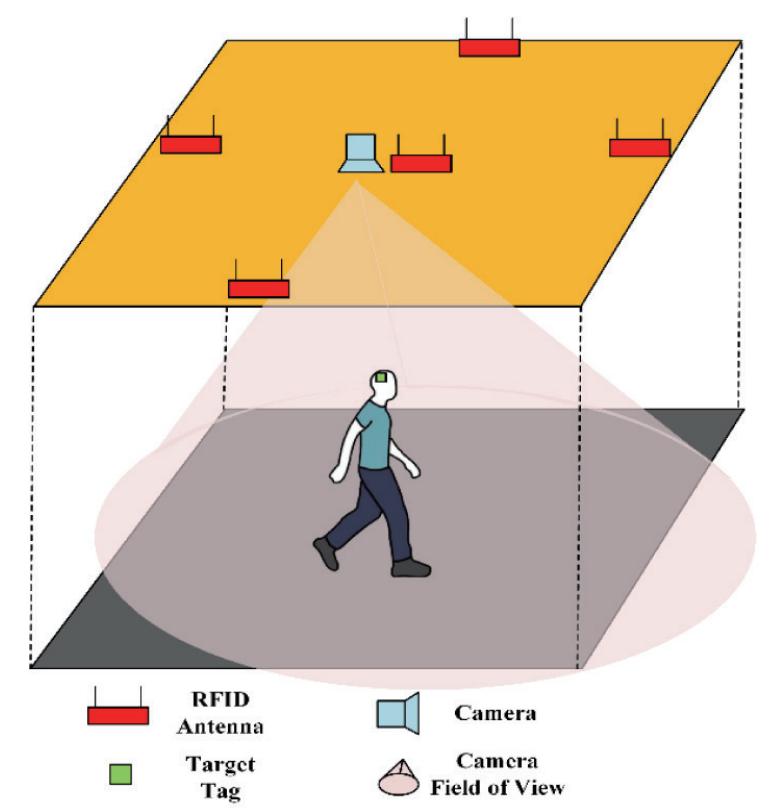

Fig. 3. (Color online) Experimental setup. 


\subsection{Experimental scenarios}

The computer vision system is used to provide the actual location of a target during the offline stage of localization to find the ideal deployment locations of RFID reader antennas. For the candidate deployment locations of the reader antennas, location fingerprints are recorded as well. To find the ideal deployment locations, the genetic algorithm determines the deployment locations of reader antennas according to the difference between the localization result of the localization algorithm and the actual location of the target. The best deployment set of locations has minimal localization error. In this study, each individual in the population is a set of 3 antennas that are selected randomly from 5 candidate antennas. Finally, the computer vision equipment can be removed after the offline stage of fingerprinting-based localization.

\section{Experimental Results}

During the offline stage of localization, the genetic algorithm searches for the locations of antenna deployment. The antenna set is selected if it has the lowest localization error. As the result of evolution (Fig. 4), the antenna set $\{\# 1, \# 3, \# 4\}$ was selected by the genetic algorithm because of its maximum number of selections. When the genetic algorithm terminates, the remaining individuals of the last generation are the survivors. For an individual, a higher number of selections indicates a greater ability to resist RSS fluctuation during the evolution of each generation. Hence, the best of three survivors are fitter individuals (antenna set) among the individuals of each generation, as shown in Fig. 4. Although the worst three individuals in the last generation also have low fitness values, their low number of selections indicate a weak ability to resist RSS fluctuations in some situations. Moreover, the localization precision is described as the cumulative distribution function (CDF), as shown in Fig. 5. The probability of the error distance lying within $0.42 \mathrm{~m}$ is 97.4\%. The experimental results show that the proposed sensor deployment method is effective in improving the accuracy of wireless indoor localization.

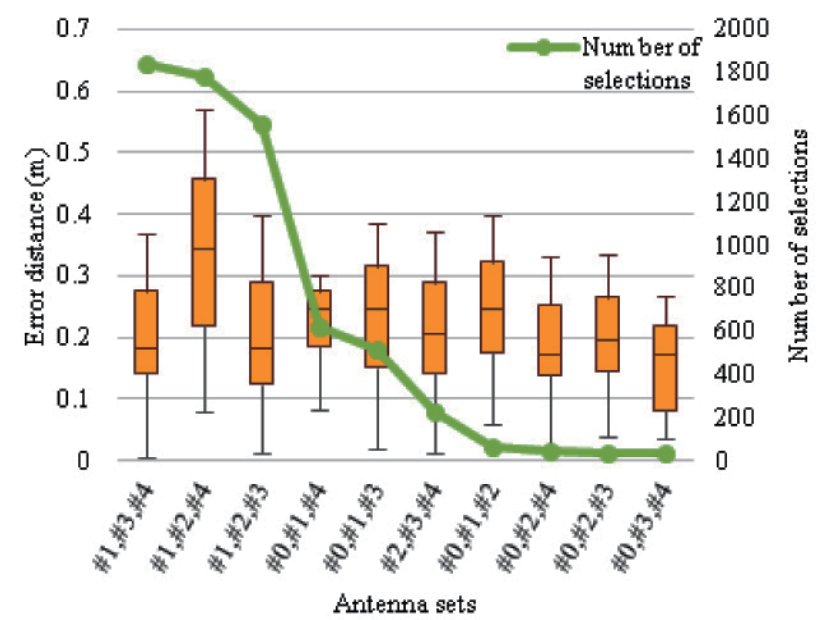

Fig. 4. (Color online) Evolution result of antenna selection.

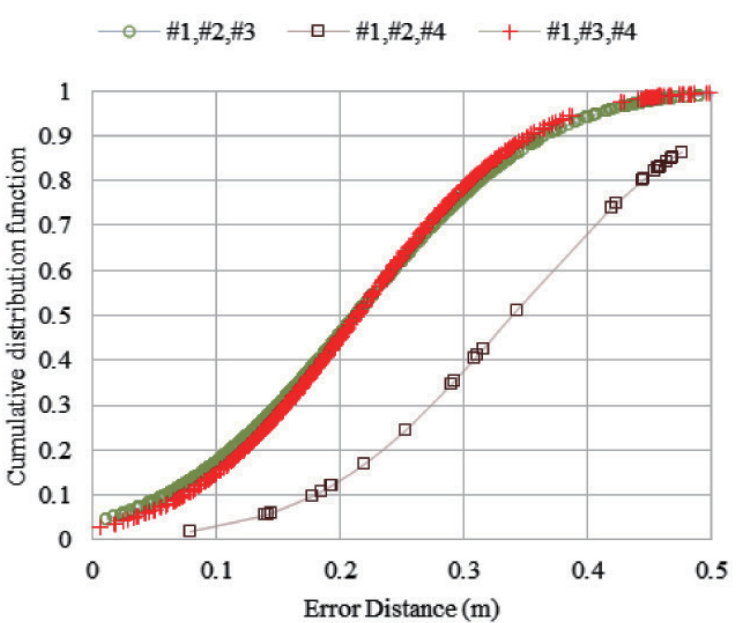

Fig. 5. (Color online) Localization precision. 


\section{Conclusions}

To construct an accurate wireless indoor localization system, the effects of environment and a moving human body need be considered. However, it is difficult to build a radio propagation model to duplicate such a complex and variable environment. In this paper, we proposed a novel sensor deployment method that combines computer vision technology and a genetic algorithm to find the optimal deployment locations of RFID reader antennas. Through the appropriate deployment of RFID reader antennas, a more realistic radio map can be provided for the use of location estimation by the fingerprinting-based localization algorithm. The proposed sensor deployment method has been implemented and evaluated. The results show that it is effective in improving the accuracy of RFID-based indoor localization.

\section{Acknowledgements}

This work was supported in part by the Ministry of Science and Technology under Grant No. MOST 104-2221-E-027-041.

\section{References}

1 K. Finkenzeller: RFID Handbook: Fundamentals and Applications in Contactless Smart Cards, Radio Frequency Identification and Near-Field Communication, 3rd ed. (Wiley, New York, 2010).

2 C. Occhiuzzi, C. Vallese, S. Amendola, S. Manzari, and G. Marrocco: Procedia Comput. Sci. 32 (2014) 190.

3 L. Catarinucci, D. de Donno, L. Mainetti, L. Palano, L. Patrono, M. L. Stefanizzi, and L. Tarricone: IEEE Internet Things J. 2 (2015) 515.

4 M. Hazas, J. Scott, and J. Krumm: IEEE Comput. 37 (2004) 95.

5 H. Liu, H. Darabi, P. Banerjee, and J. Liu: IEEE Trans. Syst. Man Cybern. Part C-Appl. Rev. 37 (2007) 1067.

6 F. Seco, A. R. Jiménez, C. Prieto, J. Roa, and K. Koutsou: Proc. IEEE Int. Symp. Intelligent Signal Processing (2009) p. 9.

7 R. Guzmán-Quirós, A. Martínez-Sala, J. L. Gómez-Tornero, and J. García-Haro: Sensors 16 (2016) 1.

8 K. Chawla and G. Robins: Int. J. Radio Freq. Identif. Technol. Appl. 3 (2011) 2.

9 S. Azzouzi, M. Cremer, U. Dettmar, T. Knie, and R. Kronberger: Proc. RFID-Technologies and Applications (IEEE, 2011) p. 174.

10 A. A. N. Shirehjini, A. Yassine, and S. Shirmohammadi: IEEE Trans. Inf. Technol. Biomed. 16 (2012) 1058.

11 S. Y. Seidel and T. S. Rappaport: IEEE Trans. Antennas Propag. 40 (1992) 207.

12 A. Bekkali, S. Zou, A. Kadri, M. J. Crisp, and R. V. Penty: IEEE Trans. Wireless Commun. 14 (2015) 1421.

13 K. Whitehouse, C. Karlof, and D. Culler: ACM SIGMOBILE Mob. Comput. Commun. Rev. 11 (2007) 41.

14 J. V. Stoep: Design and Implementation of Reliable Localization Algorithms using Received Signal Strength, MSc Thesis, University of Washington (2009).

15 P. Bahl and V. N. Padmanabhan: Proc. IEEE INFOCOM (2000) p. 775.

16 K. Kaemarungsi and P. Krishnamurthy: Proc. MobiQuitou (IEEE, 2004) p. 14.

17 J. Hightower and G. Borriello: IEEE Comput. 34 (2001) 57.

18 K. Kaemarungsi and P. Krishnamurthy: Proc. IEEE INFOCOM (2004) p. 1012.

19 Y. He, W. Meng, L. Ma, and Z. Deng: Proc. Int. ICST Conf. Communications and Networking in China (IEEE, 2011) p. 268.

20 W. Meng, Y. He, Z. Deng, and C. Li: Proc. Wireless Communications and Networking Conf. (IEEE, 2012) p. 2457.

21 Q. Wu, N. S. V. Rao, X. Du, S. S. Iyengar, and V. K. Vaishnavi: Comput. Commun. 30 (2007) 2721.

22 D. A. Coley: An Introduction to Genetic Algorithms for Scientists and Engineers (World Scientific, New Jersey, 1999).

23 Z. Michalewicz: Genetic algorithms + data structures = evolution programs, 3rd ed. (Springer, Berlin, 1996).

24 A. Moraglio, Y.-H. Kim, Y. Yoon, and B.-R. Moon: Evol. Comput. 15 (2007) 445.

25 Y. Yoon and Y.-H. Kim: Bio-Inspired Computational Algorithms and Their Applications, ed. S. Gao (InTech, Online, 2012) Chap. 4. 